\title{
CD70 Positive
}

National Cancer Institute

\section{Source}

National Cancer Institute. CD70 Positive. NCI Thesaurus. Code C132239.

Indicates that CD70 expression has been detected in a sample. 